\title{
Adolescent and Contraceptive Practices
}

\section{Matan Elami-Suzin and Joseph G. Schenker*}

Department of Obstetrics and Gynecology, Hebrew University - Hadassah Medical Centre, Jerusalem, Israel

Abstract
Adolescent pregnancy is a medical and social problem.
Sexually active adolescents are more likely to seek contraception if they perceive pregnancy as a negative
outcome, have long-term educational goals, are older, experience a pregnancy scare or actual pregnancy,
or have family, friends, and/or a clinician who encourage the use of contraception. Barriers that may
impede adolescent access to contraceptive services include lack of access to confidential services and
concerns about side effects. Before initiating contraception, it is important to review the adolescent's
history for absolute or relative contraindications, discuss the risks and benefits, and obtain consent.

Publication History:

Received: January 15, 2016

Accepted: May 17, 2016

Published: May 19, 2016

\section{Keywords:}

Noninvasive prenatal testing, Prenatal invasive fetal karyotyping

\section{Introduction}

Choosing a contraceptive method in adolescents may not be an easy task. The features of an ideal contraceptive method would be that it is: cheap; highly effective; have no side effects; independent of intercourse; does not require a regular action on the part of the user; is acceptable to all cultures and religions, and; is easily distributed and administered by non-healthcare personnel.

There is no one method that fits all of these criteria and one must choose from a variety of available methods - each with its own advantages and disadvantages. Female teens perceive cost, lack of information and lack of access to confidential healthcare services, as significant barriers. Additional barriers include: concerns regarding potential pelvic examination and fear from side effects.

\section{Oral Contraceptives}

Especially in the adolescent age group there are various adherence problems with OC's:

1. Not refilling the prescription

2. Forgetting to take the pill

3. Starting next pack late

4. Using pills inconsistently and sporadically

5. Not using a back-up method when needed

Therefore, the adolescent should be provided clear verbal and written instructions. The clinician should ascertain that she has sufficient reading skills to interpret labels and instructions.

To optimize the adolescent's adherence to OC's, we suggest giving her only the following to remember:

1. When to start the pill

2. Take the pill every day at the same time, especially when doing something else regularly, like teeth brushing

3. Call the clinic if there are any questions

OCs can be started at any time. In adolescents, they are typically started on the first day of the next menstrual period or the Sunday after the onset of the menstrual period ("Sunday start method").
The rationale for this delayed start date is to make sure that the adolescent is not pregnant. However, as many as 25 percent of adolescents who seek OCs from family planning clinics never take the first pill $[1,2]$.

It's also, in our opinion, important to give teenage girls the regiments with less hormone free interval (i.e. 24/4 preparations instead of 21/7) because these preparations allows the adolescent to simply take a pill every day and to start the next pack immediately after finishing the previous one. This makes things easy on her and she will be less likely to forget.

\section{Extended-Cycle or Continuous Pill Use}

The desire to avoid monthly periods may be related to participation in athletic events or summer camps, or to the general discomfort and "hassle" of monthly periods. A schedule that involves continuous pill use for 84 days followed by a week of pill-free days may help to increase adherence in adolescents who wish to avoid a monthly period. In addition, an oral contraceptive that provides continuous, year-round contraception has been approved by the FDA. It contains levonorgestrel $90 \mathrm{mcg}$ and ethinyl estradiol $20 \mathrm{mcg}$.

Until studies are conducted in adolescents, information on extended-cycle and continuous, year-round pills must be drawn from studies and clinical experience in adults. One-half to two-thirds of adult women report a decrease in breakthrough spotting or bleeding (BTB) in the second half compared with the first half of the extended or year-long regimen. To manage BTB, a three-day, hormone-free interval was more beneficial than continuous pills [3].

A systematic review of continuous use of oral contraceptives in adult women found that other menstrual symptoms, including headaches, genital irritation, tiredness, bloating, and dysmenorrhea also improved with extended-cycle pill use [4].

Adolescents have specific fears and concerns regarding use of contraception because of following potential side effects.

"Corresponding Author: Dr. Joseph G. Schenker, Department of Obstetrics and Gynecology. Hebrew University - Hadassah Medical Centre, Jerusalem, Israel; E-mail: joseph.schenker@mail.huji.ac.il

Citation: Elami-Suzin M, Schenker JG (2016) Adolescent and Contraceptive Practices. Int J Gynecol Clin Pract 3: 117. doi: http://dx.doi.org/10.15344/23944986/2016/117

Copyright: () 2016 Elami-Suzin et al. This is an open-access article distributed under the terms of the Creative Commons Attribution License, which permits unrestricted use, distribution, and reproduction in any medium, provided the original author and source are credited. 
Citation: Eckmann-Scholz C, Berninghaus C, Farrokh A, Maass N, Alkatotut I (2016) Prenatal Invasive Fetal Karyotyping in the Era of First Trimester Screening and Noninvasive Prenatal Testing (NIPT). Int J Gynecol Clin Pract 3: 116. doi: http://dx.doi.org/10.15344/2394-4986/2016/116

Page 2 of 7

Weight Gain - Many teenagers fear of gaining weight with the use of hormonal contraception. However, they should be reassured that available evidence does not support this regarding OC's. In the case of depot medroxyprogesterone acetate (DMPA), weight gain might be a concomitant event, but no causal relationship has been established. Factors other than DMPA may contribute to weight gain.

Bone Density - ultra-low dose estrogen (20mcg) OC's and DMPA might interfere with achieving peak bone mass in teenagers and young adults. Rare cases of osteoporosis, including fractures, have been reported in patients taking DMPA, and the prescribing information for DMPA includes a boxed warning that DMPA should be used as a long-term birth control method (e.g., longer than two years) only if other birth control methods are inadequate [5].

It is vital to share information with the adolescent regarding the potential effects of DMPA on bone density. There is a general agreement, that DMPA should be avoided early puberty, but there is lack of evidence to limit its use in older adolescents. In fact, some experts argue that for adolescents in whom DMPA is the only acceptable contraceptive option, the benefit of pregnancy prevention outweighs the potential risk to bone health.

The Society for Adolescent Health and Medicine has published a position paper regarding the boxed warning for DMPA [6]. This paper suggest that:

- With adequate explanation of benefits and potential risks, DMPA may continue to be prescribed to adolescent girls who need contraception.

- Decisions regarding bone density monitoring of adolescents using DMPA for contraception should be individualized; the decisions should be made by the clinician in concert with the adolescent, and potentially the adolescent's guardian.

- Duration of use of DMPA need not be restricted to two years.

- $\quad$ Adolescents using DMPA should be encouraged to take 1300 $\mathrm{mg}$ of elemental calcium and 400 international units vitamin D and to exercise each day.

- Estrogen supplementation should be considered for girls who are doing well on DMPA and have osteopenia or are at risk for osteopenia and who have no contraindications to estrogen.

Thromboembolism - probably one of the most feared negative consequence of combined estrogen-progestin hormonal contraception is venous thromboembolism (VTE). Use of combined OC's increases VTE risk by 2-4 fold compared to nonusers. However, this should be put readily in perspective, because the absolute risk is still very low (increases from 2-3:100oo nonusers to 8-12:1000o current users) and much lower than the VTE risk during pregnancy and postpartum period (60-80:100oo). It is, indeed, safer to prevent an unwanted pregnancy than terminating or continuing carrying it! Although reducing the estrogen dose had improved the safety and side-effects profile of OC's, it did not eliminate completely the increased risk of VTE [7-9]. There are several factors which influence the risk:

- Progestin type: Several studies and meta-analyses have reported a greater VTE risk with some of the third-generation OCs (desogestrel and gestodene, but not norgestimate) when compared with levonorgestrel, a second-generation progestin.
- In addition, two anti-androgenic progestins (drospirenone and cyproterone acetate) have been associated with a similar increase in VTE risk. However, the absolute excess risk is small, and is considered medically acceptable given the many benefits of combined hormonal contraceptives. In 2011, the European Medicines Agency also concluded that drospirenone-containing birth control pills carry a higher risk of VTE, but noted the overall risk of blood clot from any birth control method remains small and stopped short of advising women to stop taking pills containing drospirenone [10].

Acquired resistance to activated protein $\mathrm{C}$ has been suggested as one mechanism by which the third-generation OCs could predispose to VTE $[11,12]$. In addition, third-generation and second-generation progestins have different effects on the anticoagulant pathway $[13,14]$ It has been suggested that second-generation progestins, such as levonorgestrel, are more effective than third-generation progestins, such as desogestrel, in counteracting the thrombotic effects of estrogen [15].

- $\quad$ Obesity - Among OC users in two case-control studies, obese women had a 2 [16] to 24-fold [17] higher risk of VTE than nonobese women. In a third case-control study of 754 consecutive patients with a first episode of objectively diagnosed deep vein thrombosis (DVT), the risk of thrombosis among those with both obesity and OC use was increased 10-fold compared with women with neither risk factor; the increase in risk was lower (2.3 to 4.6 -fold) with either risk factor alone [18].

- $\quad$ Age - In a pooled analysis of two case-control studies, the risk of VTE rose sharply after age 39 years among women taking estrogen-progestin OCs. The estimated incidence in women over age 39 years, when compared with adolescents, was 100 versus 25 cases of VTE per 100,000 person-years, respectively [19].

- According to this analysis, the risk of VTE in adolescents who use OC's is actually the same as for non-users.

\section{Transdermal Contraceptive Patch}

Data are conflicting on whether the risk of VTE is higher in users of the transdermal contraceptive patch (Ortho Evra) than users of OC's.

Oral and injectable progestin

Oral and injectable progestin-only preparations for contraception do not appear to be associated with an increased risk of thromboembolism.

In order to preventthromboembolism, it is important to take a comprehensive and thorough history from the adolescent regarding personal and family history of VTE, for risk-stratification purposes.

- Pelvic inflammatory disease (PID) - The relationship between oral contraceptives (OCs) and pelvic inflammatory disease (PID) is complex. Whereas some studies indicate that the use of OC's increases the risk of PID, others suggest that OC's increase the risk for cervicitis and endometritis, but not salpingitis, thereby decreasing the severity of PID [20-23]. No association was found between DMPA and PID.24.

\section{Intrauterine Device}

Usually, most physicians are reluctant to insert an IUD to an adolescent, and even to nulliparous adult patient. This practice stems from several reasons, including: [25] history of negative publicity; disinformation about the risk of ectopic pregnancy, infection and 
Citation: Eckmann-Scholz C, Berninghaus C, Farrokh A, Maass N, Alkatotut I (2016) Prenatal Invasive Fetal Karyotyping in the Era of First Trimester Screening and Noninvasive Prenatal Testing (NIPT). Int J Gynecol Clin Pract 3: 116. doi: http://dx.doi.org/10.15344/2394-4986/2016/116

Page 3 of 7

and infertility; misconceptions about the mechanism of action of the IUD; lack of provider training; and fears of litigation.

In the 1970s, the IUD was used by 10 percent of women in the United States using contraception. When the Dalkon Shield (a common IUD used in that era) was linked to pelvic inflammatory disease, utilization of all IUDs fell and litigation rose.

One probable explanation for the increases infectious morbidity in the 1970's is the construction of the IUD tail, which were multifilament and braided and acted like "ropes" that allowed bacteria to ascend upward from the lower genital tract. Coupled with the fact that no test had yet been developed to detect asymptomatic Chlamydia infection. Current IUD's, which have monofilament tail strings, have not been associated with an elevated infection risk, beyond the first 2-3 weeks after insertion. In 2005, the US Food and Drug Administration (FDA) approved liberalized package labeling for the copper T380A (Paragard), removing any proscription against its use in nulliparous women or in those with more than one sexual partner. Initial studies with the newest levonorgestrel releasing IUD (LNg14 IUD; Jaydess $^{\star}$, Skyla $^{\star}$ ). Include nulliparous women. Based on the results of these trials, the product label contains no recommendation against use in nulliparous women. Although the LNg20 IUD (Mirena*) package label contains a recommendation for its use in parous women, the LNg20 has been studied in nulliparous women and found to be safe. The American College of Obstetrics and Gynecologists (ACOG) states that IUDs are safe and appropriate for most women, including nulliparous women and adolescents, and that use of IUDs should be encouraged as a first-line approach to pregnancy prevention [26,27]. The IUD is an attractive option for adolescents and adolescent mothers who desire long-term, uninterrupted contraception. It lasts 3 to 10 years depending on the type of device. Parous adolescents may be better candidates for the IUD than nulliparous adolescents because higher expulsion rates have been reported in nulliparous adolescents. However the three- and five-year devices have been successfully used in nulliparous adolescents. This has been shown in a large study based on medical records of 1177 female teenagers age 13-24 years [28]. In this study the first-attempt success rate was $95.8 \%$ for nulliparous women and $96.7 \%$ for parous women. The first-attempt success rate was $95.5 \%(n=169)$ for women aged $13-17$ years compared with $96.3 \%$ $(n=963)$ for women aged 18-24 years.

The World Health Organization suggests [29]:

- The IUD is an unacceptable risk in a woman with pelvic inflammatory disease (PID) or purulent cervicitis currently or in the past three months.

- The risks outweigh the advantages of inserting an IUD in a woman with multiple partners or a partner with multiple partners.

- There is no restriction on IUD placement when there is a past history of PID and no current sexually transmitted infection.

\section{Mechanisms of Action}

There are several possible mechanisms of action for IUD 30304, including changes in cervical mucus that inhibit sperm transport (e.g., increased copper concentration, thickening, glandular atrophy or decidualization); Chronic inflammatory changes of the endometrium and fallopian tubes, which have spermicidal effects and inhibit fertilization and implantation; Thinning and glandular atrophy of the endometrium, which inhibits implantation; and direct toxic effects on the oocyte. It is important to stress that the IUD is not an abortifacient (defined as interruption of an implanted pregnancy) [30].

\section{Absolute and Relative Contraindications}

- Absolute contraindications for any IUD placement include: Possible or confirmed pregnancy; Severe distortion of the uterine cavity (such as by fibroids or anatomic anomalies) that precludes IUD insertion;

- Acute, recent (within 3 months) or recurrent uterine infection (includes sexually transmitted, postpartum and post-abortion infections); Untreated cervicitis; Active genital actinomycoses.

- Absolute contraindications for copper IU include Wilson's disease and known copper allergy.

- Absolute contraindications for levonorgestrel IUD include: known allergy to levnorgestrel; Acute liver disease or liver tumor; and known or suspected carcinoma of the breast.

- Relative contraindications for any IUD placement include: Patients at high risk for STI's (multiple sexual partners, past infections); Previous IUD problem (perforation, significant cramps); unresolved abnormal uterine bleeding; known severe vasovagal reactions.

- Relative contraindications for copper IUD include current anemia or menorrhagia-associated anemia.

- Relative contraindications for levonorgestrel IUD include past intolerance to progestin and patient unwilling to accept a change in menstrual pattern (hypomenorrhea or amenorrhea).

\section{Complications}

\section{Uterine perforation}

This occurs in about 1:1000 interval IUD insertions. The risk factors include cervical stenosis, inexperienced provider, postpartum period, breastfeeding and fixed or retroverted uterus. If perforation by the sound or the IUD is suspected, the adolescent must be monitored for tachycardia, hypotension, syncope, or an acute abdomen. Even though these rarely occur after perforation, they warrant a transfer to hospital for further management. If perforation is suspected in a stable patient (which usually is the case), IUD removal can be attempted by placing gentle traction on the string. If the string is not visible, the location of the IUD can usually be determined by ultrasound examination.

Depending on the location, the IUD can then be retrieved using a hysteroscope or laparoscope. Removing the IUD under direct vision helps prevent injury to pelvic organs that could be snagged by the device. Laparotomy is required if laparoscopic or hysteroscopic removal is difficult, if perforation of intraabdominal organs is suspected or with ongoing intraperitoneal hemorrhage. Immediate intervention is not essential if the patient is stable. A uterine perforation caused by a sound or an IUD is not a contraindication for future vaginal delivery.

\section{Vasovagal Response}

Sometimes, the IUD insertion causes transient syncope or presyncope, hypotension, bradycardia, and/or nausea. These typically resolve by stopping the procedure, placing the adolescent in a recumbent position, and giving supportive care. 
Citation: Eckmann-Scholz C, Berninghaus C, Farrokh A, Maass N, Alkatotut I (2016) Prenatal Invasive Fetal Karyotyping in the Era of First Trimester Screening and Noninvasive Prenatal Testing (NIPT). Int J Gynecol Clin Pract 3: 116. doi: http://dx.doi.org/10.15344/2394-4986/2016/116

Page 4 of 7

\section{Post insertion patient counseling}

We teach the adolescent to feel the IUD strings and to verify periodically and especially after the menses that the IUD is retained. We guide her, that if she doesn't feel the strings, she should schedule an appointment and use a back-up contraceptive method until her healthcare provider confirms whether or not the IUD is in place. Even with absent problems we schedule a follow-up visit in 1-3 months, because it was shown to increase continuation of the method.

\section{Contraceptive Progestin Implant}

These were also suggested (together with the IUD) as a first-line contraceptive method in adolescents [26,31]. The contraceptive implant is potentially an attractive option for adolescents and adolescent mothers who desire long-term contraception. It provides pregnancy protection within 24 hours of insertion, and fertility returns quickly after its removal. Unexpected and prolonged bleeding can be a problem and can trigger request for premature removal. One should reliably exclude pregnancy before implant insertion.

\section{Uterine perforation}

Typically, the first injection is given during the menstrual period to ensure absence of pregnancy. If DMPA is initiated more than seven days after the first day of the menstrual period and she has had unprotected intercourse during the cycle, clinicians and patients must recognize that there is a small chance of preimplantation or early pregnancy despite a negative pregnancy test prior to the injection. We suggest that in these cases to receive emergency contraception if intercourse occurred within the previous 120 hours, and we advise to use back-up contraception for seven days after DMPA injection since ovulation may occur within 24 hours of the initial injection, and we counsel them to repeat the pregnancy test in 2-3 weeks.

Adolescents who are late for injection shouldn't be turned away solely because of that. After a $150 \mathrm{mg}$ injection, ovulation does not occur for at least 14 weeks. Therefore, a two-week "grace period" (repeat injection without pregnancy testing) is appropriate for adolescent women receiving injections every 3 months (13 weeks). For adolescents more than two weeks late for their injection $(>15$ weeks from the last injection), we suggest a pregnancy test before administering DMPA and back-up contraception (or abstinence) for seven days.

As a result of a study showing that contraceptive efficacy is maintained with a "grace period" as long as 4 weeks, [32] the World Health Organization (WHO) adopted a longer grace period in its updated guidelines. A limitation of these data is that more than one third of the women in the study were lactating, placing them at low baseline risk for conception.

Therefore, in high-resource countries, we continue to encourage women to return on time and allow a two-week grace period, and require documentation of a negative pregnancy test in women who present more than two weeks ( $>15$ weeks from their last injection).

\section{Pros and Cons of DMPA injections}

- Advantages ofDMPA injections include: reversibility, privacy, lasts 12 weeks, effective in obese adolescents, reduces risk of ectopic pregnancy, menses decreases or ceases, reduces dysmenorrhea and other menstrual symptoms, few drug interactions, less seizures in epileptics, less pain crises in adolescents with sickle cell anemia, reduction in endometriosis related pelvic pain, reduction in abnormal uterine bleeding associated with leiomyomas, possible reduction in the incidence of PID.

- Disadvantages of DMPA injections include: Increase in unscheduled bleeding, possible increase in weight gain, possible induction of depression, a decrease in bone density that is usually reversible, small risk of severe allergic reaction, a delay in return to fertility, repeated injection is needed every 12 weeks.

\section{Vaginal contraceptive ring}

For some adolescents this is a very attractive option because it needs to be changed only every 3 weeks. However, some adolescents do not feel comfortable in insertion of things into their vaginas. Those who use tampons might be better candidates for this method.

If the ring remains in place more than 3 but $\leq 5$ weeks, it is removed and a new one is inserted after a one-week ring-free interval; if the ring is left in place for $>5$ weeks, back-up contraception is recommended until a new ring has been in place for seven days. The ring remains effective when left in place for up to five weeks, as the ring contains sufficient steroids to maintain stable blood concentrations for this period of time [33]. Thus, inhibition of ovulation is sufficiently maintained if an adolescent forgets to remove the ring after the usual three-week use period. Adolescents who desire fewer days of withdrawal bleeding and are willing to tolerate some spotting can safely use an extended ring regimen whereby the ring is changed every three weeks, omitting the hormone-free week for up to one year [34-38]. Extended use is effective and does not worsen bothersome side effects, except for unscheduled bleeding.

Unplanned removal or expulsion of the contraceptive ring can occur. The ring should not be removed during intercourse. If it accidentally falls out, it may be rinsed with cool or warm (not hot) water and reinserted into the vagina within three hours. From our own experience, many teenagers do not feel "clean" with the vaginal ring and have a need to wash it frequently. Frequent washing decreases effectiveness and may cause intermittent bleeding or spotting.

If the ring is out of the vagina for more than three consecutive hours, subsequent steps depend on the week of the cycle that the ring is out:

- During the first two weeks of the cycle, the ring is reinserted as soon as possible. Pregnancy may not be prevented if the ring is out for more than three hours during this time, so an additional form of contraception, such as condoms with spermicide, is used until the reinserted ring has been in place for seven continuous days. The ring should subsequently be removed according to the original schedule, after which the adolescent can expect to have her normal period.

- During week three of the cycle, the adolescent discards the ring. She then chooses one of two different restart options:

- Option one - Insert a new ring and begin a new three-week cycle. Back-up contraception or abstinence is recommended until the new ring has been used continuously for seven days. The adolescent may not have a regular period until she reaches her next ring-free week, but she may have vaginal spotting or bleeding prior to that point. 
Citation: Eckmann-Scholz C, Berninghaus C, Farrokh A, Maass N, Alkatotut I (2016) Prenatal Invasive Fetal Karyotyping in the Era of First Trimester Screening and Noninvasive Prenatal Testing (NIPT). Int J Gynecol Clin Pract 3: 116. doi: http://dx.doi.org/10.15344/2394-4986/2016/116

Page 5 of 7

- Option two - Insert a new ring and begin a new three-week cycle. Back-up contraception or abstinence is recommended until the new ring has been used continuously for seven days. The adolescent may not have a regular period until she reaches her next ring-free week, but she may have vaginal spotting or bleeding prior to that point.

These steps are consistent with recommendations from the manufacturer.

\section{Transdermal patch}

The fact that the transdermal patch does not require daily attention makes it an attractive option for adolescents. However, the need for weekly change also may promote decreased adherence. The patch is applied to the buttock, abdomen, upper arm, or upper torso (but not the breast, as it might cause breast tenderness due to high local estrogen concentration). A different site is used each time a new patch is applied. The patch is changed once a week for three weeks (21 total days), followed by one week that is patch-free. It should always be changed/ applied on the same day of the week.

Reminder systems are useful to ensure appropriate weekly changes. The consequences of failing to change the transdermal contraceptive patch at the appropriate time should be addressed with the adolescent.

- Delay in beginning the first patch in a cycle - When a new patch cycle is delayed beyond the scheduled start day, users are instructed to apply a patch as soon as they remember and use back-up contraception (or avoid sex) for at least one week. The day they apply the new patch becomes the new patch change day.

- Delay in beginning the second or third patch in a cycle - There is a two-day (48 hours) period of continued release of adequate contraceptive steroid levels when the patch is left on for two extra days. If users change the patch within this window, the patch change day remains the same and there is no need for back-up contraception.

After this two-day (48 hours) time period, failure to replace the second or third patch in a cycle increases the risk for contraceptive failure. Therefore, users will need to use back-up contraception (or avoid sex) for seven days and, in some instances, use emergency contraception, if this occurs. The day the patient remembers to apply the patch becomes the new change day.

- Delay in removing the third patch in a cycle - Forgetting to remove the third patch on time carries less risk than forgetting to remove the first or second patch. The user is instructed to remove the patch when she remembers; the patch change day is not altered.

Strategies to promote weekly changes should be reviewed with adolescents using the transdermal patch. These include using a wall, computer, or cell phone calendar; a cell phone weekly alarm; or a sticker designating the change day on the bathroom mirror.

If a patch becomes partially or completely detached for less than 24 hours, it should be reapplied at the same location (if it has not lost its stickiness: ancillary adhesives or tape should not be used), or replaced with a new patch immediately. If detachment lasts longer than 24 hours, a new patch should be applied, and this day of the week becomes the new patch change day. An additional method of contraception (e.g., condoms, spermicides) should be used for the first seven days of this cycle or the patient should avoid sex.

In various trials, 1.8 percent of transdermal patches required replacement for complete detachment and 2.9 percent became partially detached [39]. Living in a warm, humid climate did not increase the risk of detachment. The quality of adherence was illustrated in a study in which 30 women were subjected to various conditions over several seven-day time periods during transdermal patch use [39]. The conditions included normal activity, use of a sauna, immersion in a whirlpool bath, use of a treadmill followed by showering, cool water immersion, and a combination of these activities. Only one patch became detached during the 87 cycles that were evaluated, suggesting that skin adherence is not adversely affected by a vigorous, athletic lifestyle.

\section{Condoms}

We always stress the benefit of dual-protection against both pregnancy and STI's. Girls planning to use condoms should receive them before leaving the office or clinic, if possible, to ensure availability and promote adherence.

\section{Emergency Contraception (EC)}

Emergency contraception (also known as postcoital contraception and the morning-after pill) refers to the use of drugs or a device as an emergency measure to prevent pregnancy. As adolescents are particularly prone to have unprotected intercourse, they are potential candidates for EC. It is intended for occasional or back-up use, not as a primary contraceptive method for routine use.

In most developed countries today, some form of orally given EC is available as over-the-counter drug. Both oral and intrauterine EC are only effective before implantation.

\section{Orally given EC that are available}

Levonorgestrel - $0,75 \mathrm{mg}$ given twice daily 12 hours apart up to 72 hours efficiency 60 to $94 \%$

Mifepristone- single dose $600 \mathrm{mg}$, up to 5 daysefficiency 99 to $100 \%$ Ulipristal -single oral dose of 30mgup to 72 hours, efficiency 99 to $100 \%$.

It is recommended that Ulipristal acetate be avoided in women using enzyme-inducing drugs or who have taken them within the last 28 days. Patients should also be advised to avoid Ulipristal if they are currently taking drugs that increase gastric $\mathrm{pH}$ (e.g., antacids, histamine $\mathrm{H} 2$ antagonists and proton pump inhibitors).

Although the Centers for Disease Control and Prevention (CDC) and the World Health Organization's (WHO) Medical Eligibility Criteria for Contraceptive Use applies contraindications to daily use of hormonal contraceptives in some women based on their medical history, these contraindications do not apply to adolescents and women seeking emergency contraception. In particular, cardiovascular disease, thrombophilic disorders, migraine, liver disease, and breastfeeding are considered conditions where the advantages of using the method generally outweigh the theoretical or proven risks. 
Citation: Eckmann-Scholz C, Berninghaus C, Farrokh A, Maass N, Alkatotut I (2016) Prenatal Invasive Fetal Karyotyping in the Era of First Trimester Screening and Noninvasive Prenatal Testing (NIPT). Int J Gynecol Clin Pract 3: 116. doi: http://dx.doi.org/10.15344/2394-4986/2016/116

Page 6 of 7

Some data suggest levonorgestrel and ulipristal emergency contraception may be less effective in overweight or obese women. A 2015 study reported that levonorgestrel emergency contraception was less effective at preventing pregnancy as weight and body mass index increased [40]. The women in the $80 \mathrm{~kg}$ group had a pregnancy risk of 6 percent, which was similar to the pregnancy risk as if no contraceptive had been used. At least one pharmaceutical package insert for levonorgestrel emergency contraception warns of reduced contraceptive efficacy in women weighing $\geq 75 \mathrm{~kg}$. However, the European Medicines Association concluded that the available data were too limited and not robust enough to be certain the contraceptive efficacy of levonorgestrel or ullipristal emergency contraception is reduced with increased bodyweight and that the benefits of taking these medications outweigh any risks [41].

Copper intrauterine device, inserted up to 5 days, efficiency at least $99 \%$

Contraindications to copper IUD use include severe uterine distortion, active pelvic infection, copper allergy, and suspected pregnancy.

\section{Resuming or initiating hormonal contraception}

The duration of effectiveness of emergency contraception has not been determined. Thus it is not clear when regular contraception should be resumed or initiated within the menstrual cycle of emergency contraceptive use [42]. The safest approach is to advise adolescents using emergency contraception pills that a risk of pregnancy still exists if they have unprotected sexual intercourse after the emergency contraceptive pills have been taken; therefore, they should immediately initiate an effective contraceptive method.

For those taking Levonorgestrel or combined estrogen-progestin emergency contraception, any regular contraceptive method can be started immediately after the use of the emergency contraceptive [43] Backup methods of contraception (e.g., condom or diaphragm) or abstinence are required during the first seven days of use. Although efficacy is not compromised by immediate initiation, it is prudent to avoid starting longer-acting hormonal methods (e.g., depotmedroxyprogesterone acetate, etonogestrel implant, Levonorgestrelreleasing intrauterine devices) until it is certain that pregnancy has not occurred. However, this concern should be balanced against risk of unintended pregnancy if initiation of one of these methods is delayed.

For those taking Ulipristal for emergency contraception, there is theoretical concern that starting a hormonal method after taking Ulipristal could make either the Ulipristal or the hormonal method less effective by competitive binding to the progestin receptors. In 2015, the US Food and Drug Administration amended the package insert and stated that progesterone-containing contraceptives should not be used with ulipristal or for five days following ulipristal use [44]. The ulipristal package insert recommends using a barrier method for the remainder of the cycle to avoid any potential drug interactions with a hormonal contraceptive. Some experts advise waiting seven days after ulipristal to start a hormonal method. A guideline from the United Kingdom recommends that additional precautions be taken for 14 days after taking ulipristal (nine days if using or starting the progestogen-only pill, 16 days, forestradiol valerate/dienogest pill) [45].

\section{Repeated emergency contraception}

There is no contraindication to giving a second dose of hormonal emergency contraception if a second episode of unprotected intercourse occurs any time after the first dose was administered. In contrast, there is no support for use of Ulipristal more than once per cycle or if there has been another episode of unprotected sexual intercourse outside the treatment window (>120 hours) [43].

\section{Conclusion}

Adolescents are a vulnerable population in terms of unintended pregnancies and sexually transmitted infections. In this review, we have specifically regarded issues relevant to correct and successful use of different contraceptive methods in adolescents. The important "take-home" messages are:

1. We should provide counseling and ensure access for a range of contraceptive methods. We should allow sufficient time during the medical encounter to dispel myths and provide reassurance about the general safety of various contraceptive methods.

2. Age per se is not a contraindication to any type of contraceptive, and neither nulliparity.

3. The LARC methods should be considered first-line choice for adolescents.

4. There is no need for a pelvic exam before prescribing hormonal contraception in asymptomatic healthy adolescents.

5. Always encourage "dual-protection" with condom use in every sexual intercourse.

6. We should remember and stress the non-contraceptive benefits of hormonal contraception.

7. Adolescents with disabilities and chronic illnesses have sexual health and contraception need that are similar to their healthy peers.

\section{Competing Interests}

The authors declare that they have no competing interests.

\section{References}

1. Polaneczky M, Slap G, Forke C, Rappaport A, Sondheimer S (1994) The use of levonorgestrel implants (Norplant) for contraception in adolescent mothers. N Engl J Med 331: 1201-1206.

2. Lara-Torre E (2004) "Quick Start", an innovative approach to the combination oral contraceptive pill in adolescents. Is it time to make the switch? J Pediatr Adolesc Gynecol 17: 65-67.

3. Coffee A, Sulak PJ, Kuehl TJ (2007) Long-term assessment of symptomatology and satisfaction of an extended oral contraceptive regimen. Contraception 75: 444-449.

4. Edelman, Gallo MF, Nichols MD, Jensen JT, Schulz KF, et al. (2006) Continuous versus cyclic use of combined oral contraceptives for contraception: systematic Cochrane review of randomized controlled trials. Hum Reprod 21: 573-578.

5. [No authors listed] (2005) 'Black box' warning added to contraceptive injection. FDA Consum 39: 3 .

6. Cromer BA, Scholes D, Berenson A, Cundy T, Clark MK, et al. (2006) Depot medroxyprogesterone acetate and bone mineral density in adolescents-the Black Box Warning: a Position Paper of the Society for Adolescent Medicine. J Adolesc Health 39: 296-301. 
Citation: Eckmann-Scholz C, Berninghaus C, Farrokh A, Maass N, Alkatotut I (2016) Prenatal Invasive Fetal Karyotyping in the Era of First Trimester Screening and Noninvasive Prenatal Testing (NIPT). Int J Gynecol Clin Pract 3: 116. doi: http://dx.doi.org/10.15344/2394-4986/2016/116

7. Vandenbroucke JP, Rosing J, Bloemenkamp KW, Middeldorp S, Helmerhorst FM, et al. (2001) Oral contraceptives and the risk of venous thrombosis. N Engl J Med 344: 1527-1535.

8. Douketis JD, Ginsberg JS, Holbrook A, Crowther M, Duku EK, et al. (1997) A reevaluation of the risk for venous thromboembolism with the use of ora contraceptives and hormone replacement therapy. Arch Intern Med 157: 1522-1530.

9. Bloemenkamp KW, Rosendaal FR, Büller HR, Helmerhorst FM, Colly LP et al. (1999) Risk of venous thrombosis with use of current low-dose ora contraceptives is not explained by diagnostic suspicion and referral bias. Arch Intern Med 159: 65-70.

10. PhVWP (2012) Monthly report on safety concerns, guidelines and genera matters. Europ Medicin Agency.

11. Rosing J, Tans G, Nicolaes GA, Thomassen MC, van Oerle R, et al. (1997) Oral contraceptives and venous thrombosis: different sensitivities to activated protein $\mathrm{C}$ in women using second- and third-generation ora contraceptives. Br J Haematol 97:233-238.

12. Rosing J, Middeldorp S, Curvers J, Christella M, Thomassen LG, et al (1999) Low-dose oral contraceptives and acquired resistance to activated protein C: a randomised cross-over study. Lancet 354: 2036-2040.

13. Middeldorp S, Meijers JC, van den Ende AE, van Enk A, Bouma BN, et al (2000) Effects on coagulation of levonorgestrel- and desogestrel-containing low dose oral contraceptives: a cross-over study. Thromb Haemost 84: 4-8.

14. Kemmeren JM, Algra A, Meijers JC, Bouma BN, Grobbee DE (2002) Effects of second and third generation oral contraceptives and their respective progestagens on the coagulation system in the absence or presence of the factor V Leiden mutation. Thromb Haemost 87: 199-205.

15. Kemmeren JM, Algra A, Meijers JC, Tans G, Bouma BN, et al. (2004) Effect of second- and third-generation oral contraceptives on the protein $C$ system in the absence or presence of the factor VLeiden mutation: a randomized trial. Blood 103: 927-933.

16. Sidney S, Petitti DB, Soff GA, Cundiff DL, Tolan KK, et al. (2004) Venous thromboembolic disease in users of low-estrogen combined estrogenprogestin oral contraceptives. Contraception 70: 3-10.

17. Pomp ER, le Cessie S, Rosendaal FR, Doggen CJ (2007) Risk of venous thrombosis: obesity and its joint effect with oral contraceptive use and prothrombotic mutations. Br J Haematol 139: 289-296.

18. Abdollahi M, Cushman M, Rosendaal FR (2003) Obesity: risk of venous thrombosis and the interaction with coagulation factor levels and ora contraceptive use. Thromb Haemost 89: 493-498.

19. Nightingale AL, Lawrenson RA, Simpson EL, Williams TJ, MacRae KD et al. (2000) The effects of age, body mass index, smoking and genera health on the risk of venous thromboembolism in users ofcombined ora contraceptives. Eur J Contracept Reprod Health Care 5: 265-274.

20. Louv WC, Austin H, Perlman J, Alexander WJ (1989) Oral contraceptive use and the risk of chlamydial and gonococcal infections. Am J Obstet Gynecol 160: 396-402.

21. Rubin GL, Ory HW, Layde PM (1982) Oral contraceptives and pelvic inflammatory disease. Am J Obstet Gynecol 144: 630-635.

22. Ness RB, Keder LM, Soper DE, Amortegui AJ, Gluck J, et al. (1997) Ora contraception and the recognition of endometritis. Am J Obstet Gynecol 176: $580-585$.

23. Wolner-Hanssen P, Svensson L, Mardh PA, Westrom L (1985) Laparoscopic findings and contraceptive use in women with signs and symptoms suggestive of acute salpingitis. Obstet Gynecol 66: 233-238.

24. Ness RB, Soper DE, Holley RL, Peipert J, Randall H, et al. (2001) Hormonal and barrier contraception and risk of upper genital tract disease in the PID Evaluation and Clinical Health (PEACH) study. Am J Obstet Gynecol 185 121-127.

25. Stanwood NL, Garrett JM, Konrad TR (2002) Obstetrician-gynecologists and the intrauterine device: a survey of attitudes and practice. Obstet Gynecol 99: 275-280.

26. Committee on Adolescent Health Care Long-Acting Reversible Contraception Working Group TACoO (2012) Gynecologists. Committee opinion no. 539: adolescents and long-acting reversible contraception implants and intrauterine devices. Obstet Gynecol 120: 983-988.
27. ACOG Committee Opinion no. 450: Increasing use of contraceptive implants and intrauterine devices to reduce unintended pregnancy. Obstet Gynecol 114:1434-1438.

28. Teal SB, Romer SE, Goldthwaite LM, Peters MG, Kaplan DW, et al. (2015) Insertion characteristics of intrauterine devices in adolescents and young women: success, ancillary measures, and complications. Am J Obste Gynecol 213: 515.

29. Medical Eligibility Criteria for Contraceptive Use (2010) A WHO Family Planning Cornerstone. 4th ed Geneva.

30. Stanford JB, Mikolajczyk RT (2002) Mechanisms of action of intrauterine devices: update and estimation of postfertilization effects. Am J Obstet Gynecol 187: 1699-1708.

31. Ott MA, Sucato GS (2014) Committee on A. Contracep for adol Pediatric 134: e1257-1281.

32. Steiner MJ, Kwok C, Stanback J, Byamugisha JK, Chipato T, et al (2008) Injectable contraception: what should the longest interval be for reinjections? Contracept 77: 410-414.

33. Mulders TM, Dieben TO (2001) Use of the novel combined contraceptive vaginal ring NuvaRing for ovulation inhibition. Fertil Steril. 75: 865-870.

34. Barreiros FA, Guazzelli CA, Barbosa R, de Assis F, de Araujo FF (2010) Extended regimens of the contraceptive vaginal ring: evaluation of clinical aspects. Contracept 81: 223-225.

35. Miller L, Verhoeven $\mathrm{CH}$, Hout $\mathrm{J}$ (2005)Extended regimens of the contraceptive vaginal ring: a randomized trial. Obstet Gynecol $106: 473$ 482.

36. Barreiros FA, Guazzelli CA, de Araujo FF, Barbosa R (2007) Bleeding patterns of women using extended regimens of the contraceptive vaginal ring. Contraception 75: 204-208.

37. Guazzelli CA, Barreiros FA, Barbosa R, de Araujo FF, Moron AF (2009) Extended regimens of the vaginal contraceptive ring: cycle control. Contraception 80: 430-435.

38. Barreiros FA, Guazzelli CA, Barbosa R, Torloni MR, Barbieri M, et al (2011) Extended regimens of the combined contraceptive vaginal ring containing etonogestrel and ethinyl estradiol: effects on lipid metabolism. Contraception. 84: 155-159.

39. Zacur HA, Hedon B, Mansour D, Shangold GA, Fisher AC, et al. (2002) Integrated summary of Ortho Evra/Evra contraceptive patch adhesion in varied climates and conditions. Fertil Steril 77: S32-35.

40. Kapp N, Abitbol JL, Mathé H, Scherrer B, Guillard H, et al. (2015) Effect of body weight and BMI on the efficacy of levonorgestrel emergency contraception. Contraception 91: 97-104.

41. Levonorgestrel and ulipristal remain suitable emergency contraceptives for all women, regardless of bodyweight.

42. Salcedo J, Rodriguez MI, Curtis KM, Kapp N (2013) When can a woman resume or initiate contraception after taking emergency contraceptive pills? A systematic review. Contracep 87: 602-604.

43. Practice Bulletin No. 152 (2015) Emergency Contraception. Obstet Gynecol 126: e1-e11.

44. Ulipristal acetate. US FDA approved product information. National Library of Medicine.

45. Clinical Effectiveness Unit (2011) Combined hormonal contraception London (UK): Faculty of Sexual and Reproductive Healthcare. 